\title{
A phenomenological model for shape memory alloy spring based on differential hysteresis theory
}

\author{
Yang $\mathrm{Yu}^{1, \mathrm{a}}$, Abubakar R. A. ${ }^{2, b}$ and Ting $\mathrm{Wu}^{3, \mathrm{c}}$ \\ ${ }^{1}$ Department of Mechanical Engineering, Guangdong Songshan Polytechnic College, \\ Shaoguan Guangdong 512126China, \\ ${ }^{2,3}$ The Institute of mechanical design, Department of mechanical engineering, Zhejiang \\ University, China, 310027 \\ Email: 3374952649@qq.com, brbkiru@yahoo.com, `wt13816402949@126.com
}

Key word: phenomenological model; SMA spring; pseudo-elasticity; differential hysteresis Abstract: As a kind of smart material, Shape memory alloy spring has multiple uses, especially as a good potential for solving vibration isolation problem. In this paper, a phenomenological model for describing pseudo-elasticity of shape memory alloy (SMA) spring has been developed. The differential hysteresis theory is applied here and introduces bifurcation to the system which incorporates SMA spring. The Lagrangian of the system is used to derive dynamic response of SMA spring. Comparisons between the numerical results and the experimental counterparts are presented. With appropriate method of parameter identification, a perfect fit of the model results with the experimental measurements is obtained.

\section{Introduction}

For the past two decades, many mathematical model and dynamic model of shape memory alloy (SMA) has been constructed from different perspectives [1-4]. Recently SMA spring has aroused researchers' attention due to its outstanding features in the field of actuation and vibration control. Compared to SMA wire as actuator, SMA spring output a larger displacement. When SMA wire is formed as spring, it also enlightens the existing vibration isolation solution for its internal damping capability and pseudo-elasticity. However, the pursuit of modeling its dynamic process is still under spotlight. This paper will lay stress on the modeling of pseudoelasticity of SMA spring.

C. Liang first explores the applications of SMA spring in vibration control [5]. Based on his constitutive relations of SMA, he introduced the corresponding method for designing SMA spring. The modified-Preisach model proposed by D.C. Lagoudas has been applied to model the structural response of a SMA tube as a spring element [6]. Greater number of experiment data is needed to ensure the accuracy of model fitting. A linear part and an exponential part is combined to compose the phenomenological model by Bin Huang [7], although it is applicably utilized in analysis in vibration isolation system, it is less related to essence of pseudoelasticity. Inspired by those attempts, in order to construct an appropriate model for SMA spring that can capture its pseudoelasticity, a Landau-type torsional free energy is established to describe phase transformation which is dependent on temperature. Beyond austenite finish temperature, SMA spring shows pseudoelasticity that could be used in vibration control. A phenomenological 
model for SMA spring is given and comparison between experiment data and model presents great repeatability.

\section{Model of SMA spring}

By following Landau theory for phase transformation, a non-convex free energy of the chosen order parameter shall be constructed to characterize different phases involved in the system [8]. For the current model of the nonlinear dynamics of the torsional SMA wire, the torsion strain in the cross section could be used to characterize the state of the SMA wire, Therefore a modified Landau free energy function could be constructed as follows:

$$
\psi(\theta, \alpha)=\frac{k_{1}\left(\theta-\theta_{c}\right)}{2} \alpha^{2}+\frac{k_{2}}{4} \alpha^{4}+\frac{k_{3}}{6} \alpha^{6}
$$

Where $\theta$ is material temperature, $\alpha$ is the torsional strain and chosen as the only order parameter, and $k_{1}, k_{2}, k_{3} \theta_{c}$ are all material constants. Please note that in the current paper the microstructure in the SMA wire is not considered, but the hysteretic dynamics of the SMA wire as a whole. Therefore one can ignore the torsional strain distribution on the cross section of the SMA wire, and simply assume that the torsional strain is uniform in the considered SMA wire and $\alpha$ can be simply regarded as the torsion angle. It is obvious that the free energy given by Eq. (1) depends on the material temperature. As illustrated in Fig.1, three profiles of the free energy function are presented for three different temperatures. At high temperatures, only austenite is stable and there is only one stable state, no phase transformation would be induced. While at low temperature, the free energy function curve has two local minima corresponding to two martensite variants. Whilst at intermediate temperature, there are three local minima in the free energy function. The two symmetric minima are associated with the two symmetric martensite variants (marked as ellipses for $\mathrm{M}+$ and $\mathrm{M}-$ ). The local minimum at the center is associated with austenite (marked as rectangle). It is shown clearly by Fig.1 (b) that both martensite and austenite could exist in this case, it is also clearly illustrated that phase transformation would be induced when the material temperature changes because some phases would lose its stability at some temperature ranges. 


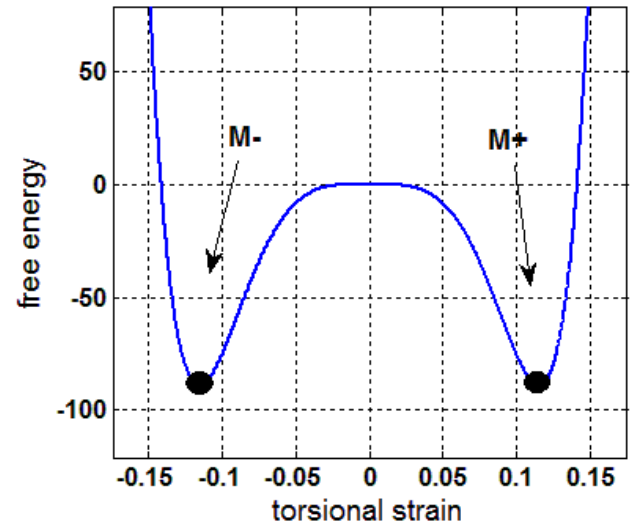

(a)

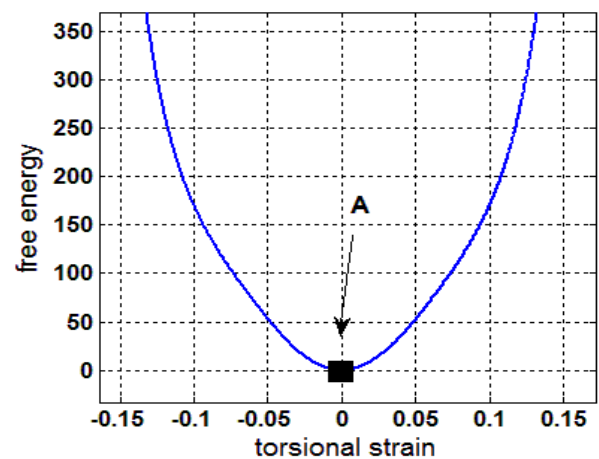

(c)

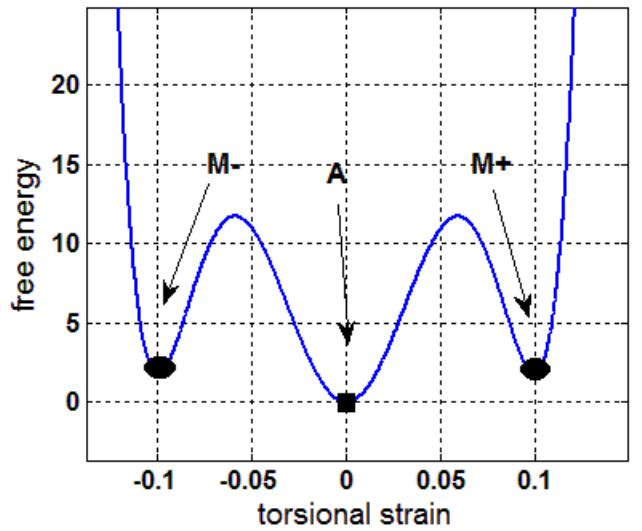

(b)

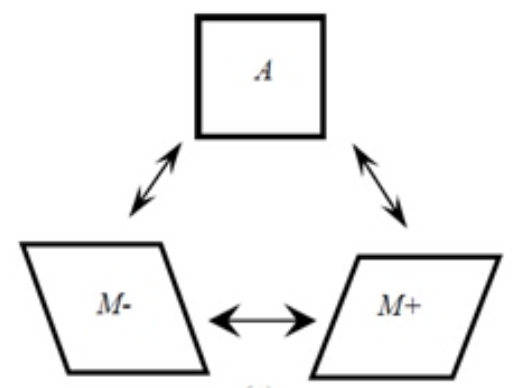

(d)

Fig.1. profiles of the free energy function at different temperatures for modeling of phase transformation under torsion: (a) Low temperature case; (b) Intermediate temperature case; (c) High temperature case; (d) Sketch of phase transformation

Using thermo-mechanical equilibrium conditions, the torque-torsion constitutive relations can be obtained from above free energy function.

$$
T=\frac{\partial \psi}{\partial \alpha}
$$

This leads to the following constitutive law:

$$
T=k_{1}\left(\theta-\theta_{c}\right) \alpha+k_{2} \alpha^{3}+k_{3} \alpha^{5} .
$$

It is obvious that the three material constants $k_{1}, k_{2}, k_{3}$ now also incorporate the influence of the geometrical parameter $r$, the radius of the SMA wire, since the torsional strain and stress is assumed to be uniform (independent on the radius).

In order to recast the above torsional stress-strain constitutive law into the force-displacement relation of a SMA spring, one need to perform the following transform [9]. When the spring is made, an external load is applied as shown in figure 2, the displacement $\mathrm{x}$ shares the following relationship with $\alpha$, which is the angular deflection of one end of the spring wire with respect to the other. 


$$
x=\alpha R \text {. }
$$

Where $\mathrm{R}$ is the radius of SMA spring. Using this assumption, analysis of the helical spring is reduced to that of the pure torsion of a straight wire. Then whenever the SMA spring reaches its equilibrium state, it is under the action of the two forces $\mathrm{F}$ and resisting torsional moment $\mathrm{T}$. and the torque equals:

$$
T=F R
$$

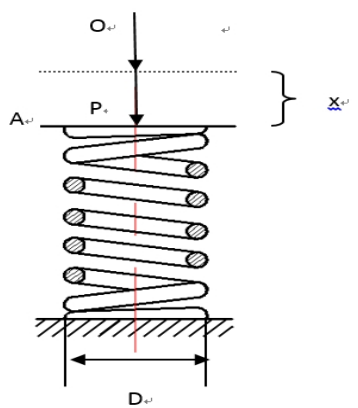

Figure 2: SMA spring under deformation

Since the relation between a straight SMA wire and a SMA spring is obtained, the constitutive relation of the spring can be derived by substitute the above relations into equation (3), thus:

$$
f=\frac{k_{1}\left(\theta-\theta_{c}\right)}{R^{2}} x+\frac{k_{2}}{R^{4}} x^{3}+\frac{k_{3}}{R^{6}} x^{5} \text {. }
$$

\section{Single crystalline model of shape memory alloy spring}

To describe the dynamics of the SMA spring in mechanical field, the Lagrangian of the SMA spring is introduced as a combination of kinetic and potential energy contributions as follows

$$
L=\int_{0}^{l}\left(\frac{\rho}{2} \&-\Psi\right) d x
$$

Where $p$ is the density of the material, $x$ is the displacement of the SMA spring, $\Psi$ is the

potential energy density of the material stored via deformations, while $\frac{\rho}{2} \dot{x}^{2}$ models the kinetic energy density.The force-displacement relationship of SMA spring is given as the following simplified form:

$$
f=a_{1}\left(\theta-\theta_{c}\right) x+a_{2} x^{3}+a_{3} x^{5} .
$$

Where the correspondence between the coefficients $a_{i}$ with those $k_{i}$ is obvious. When the SMA spring is subject to compression, a certain amount of energy is converted into the deformation of SMA spring, which can be denoted as below: 


$$
W=\int_{x}^{0}(-f x) d x
$$

In order to account for dissipation effects accompanying phase transformations, a dissipation term is introduced here as follows:

$$
R=-\frac{1}{2} v\left(\frac{d x}{d t}\right)^{2} .
$$

Where $v$ is a coefficient accounting for dissipation effects in phase transformation process. By substituting the potential energy function into Eq. (7), and using the Euler - Lagrange equation, the governing equation for the dynamics of the SMA spring can be obtained as the following:

$$
\frac{d^{2} x}{d t^{2}}+v \frac{d x}{d t}=a_{1}\left(\theta-\theta_{c}\right) x+a_{2} x^{3}+a_{3} x^{5}+f(\mathrm{t})
$$

Where $f(\mathrm{t})$ represents the external input to the system. For the current problem, the inertia effects in above differential equation is much smaller than the dissipation effect[2,9], so the second order ordinary differential equation could be simplified as the first order one as follows

$$
v \frac{d x}{d t}=A_{1} x+A_{2} x^{3}+A_{3} x^{5}+f(\mathrm{t})
$$

Where $v$ can be seen as the time constant in the current differential equation, $A_{i}$ is the new parameter incorporating temperature term, given that temperature here is a fixed value. If the loading $f(t)$ is kept as a constant (or sufficiently slow), the system will achieve its equilibrium state, which gives the static restoring force of the SMA spring ( $\operatorname{setting} \frac{d x}{d t}=0$ ):

$$
F=A_{1} x+A_{2} x^{3}+A_{3} x^{5}
$$

It can be easily illustrated that hysteresis is embedded in the dynamics given in (12), when the parameters are set appropriately. The F-x relation given in Eq. (13) is sketched in Fig.3.

\section{Result}

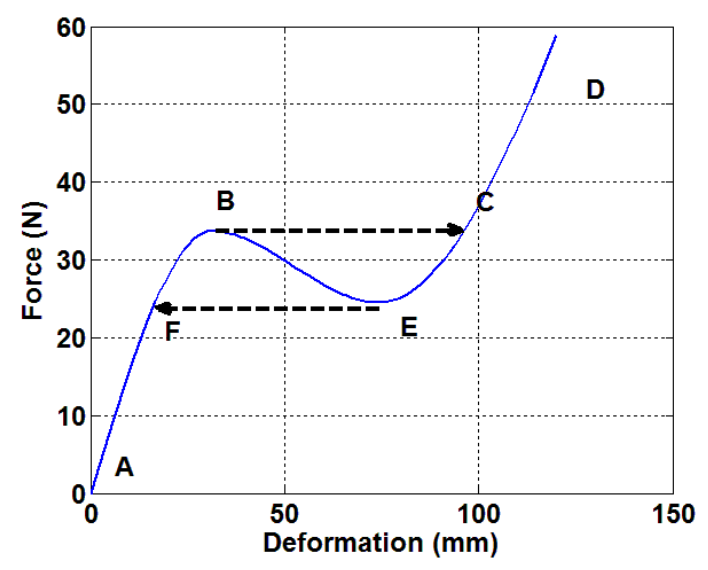

Fig.3 Constitutive curve and predicted jump phenomenon 


\section{Discussion}

It can be seen that the constitutive curve is non-monotonic, leading bifurcation to the differential Eq. (12) [10]. The dashed lines in the plot represent the predicted jump. Thus the whole dynamic process can justify the hysteresis phenomenon of SMA spring.

\section{Conclusion}

In this paper, a phenomenological model of SMA spring is constructed based on the non-convex constitutive relation of shape memory alloy. A modified Landau-type free energy function is applied to the torsion of SMA sample and can best capture the underlying phase transformation when SMA spring is loading. Pseudoelasticity of SMA spring is captured by applying constitutive relation in a Lagrangian. The polycrystalline model is constructed with the help of Cauchy distribution function. The accuracy of this dynamical differential model is proved by compared to the experimental data. It shows that the proposed model successfully captures the hysteretic dynamics of SMA spring, in a rather simple way.

\section{Acknowledgements}

This work has been supported by the National Natural Science Foundation of China (Grant No. 51575478 and Grant No. 61571007). RM acknowledges the support from the NSERC and CRC program.

\section{References}

[1] Liang, C., \& Rogers, C. A. (1990). One-dimensional thermomechanical constitutive relations for shape memory materials. Journal of intelligent material systems and structures, 1(2), 207-234.

[2] Auricchio, F., Taylor, R. L., \&Lubliner, J. (1997). Shape-memory alloys: macromodelling and numerical simulations of the superelastic behavior. Computer methods in applied mechanics and engineering, 146(3), 281-312.

[3] Savi, M. A., Paiva, A., Baeta-Neves, A. P., \& Pacheco, P. M. (2002). Phenomenological modeling and numerical simulation of shape memory alloys: a thermo-plastic-phase transformation coupled model. Journal of Intelligent Material Systems and Structures, 13(5), 261-273.

[4] Sun, S., \&Rajapakse, R. K. N. D. (2003). Simulation of pseudoelasticbehaviour of SMA under cyclic loading. Computational Materials Science, 28(3), 663-674.

[5] Liang, C., \& Rogers, C. A. (1993). Design of shape memory alloy springs with applications in vibration control. Journal of Vibration and Acoustics, 115(1), 129-135.

[6] Lagoudas, D. C., Khan, M. M., Mayes, J. J., \& Henderson, B. K. (2004). Pseudoelastic SMA spring elements for passive vibration isolation: Part II-Simulations and experimental correlations.Journal of Intelligent Material Systems and Structures, 15(6), 443-470.

[7] Huang, B., Zhang, H., Wang, H., \& Song, G. (2014). Passive base isolation with superelasticnitinol SMA helical springs. Smart Materials and Structures, 23(6), 065009. 
[8] Bubner, N. (1996). Landau-Ginzburg model for a deformation-driven experiment on shape memory alloys. Continuum Mechanics and Thermodynamics, 8(5), 293-308.

[9] Wahl, A. M. Mechanical springs, 1963.McGAWHILL, p119.

[10] Wang, L. X., \&Willatzen, M. (2007). Nonlinear dynamical model for hysteresis based on nonconvex potential energy. Journal of engineering mechanics, 133(5), 506-513.

[11] Savi, M. A., Pacheco, P. M. C., Garcia, M. S., Aguiar, R. A., de Souza, L. F. G., \& da Hora, R. B. (2015). Nonlinear geometric influence on the mechanical behavior of shape memory alloy helical springs. Smart Materials and Structures,24(3), 035012. 